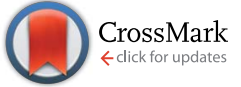

Cite this: RSC Adv., 2017, 7, 14669

Received 19th January 2017

Accepted 22nd February 2017

DOI: $10.1039 / c 7 r a 00807 d$

rsc.li/rsc-advances

\section{Pyridinic and pyrrolic nitrogen-rich ordered mesoporous carbon for efficient oxygen reduction in microbial fuel cells $\uparrow$}

\author{
Leiyu Feng, ${ }^{\text {ab }}$ Xutao Chen, ${ }^{a}$ Yue Cao, ${ }^{a}$ Yunzhi Chen, ${ }^{a}$ Feng Wang, ${ }^{a}$ Yinguang Chen ${ }^{\star a}$ \\ and Yin Liu ${ }^{\mathrm{C}}$
}

Non-noble cathode catalysts with high performance and low cost are vital for large-scale applications of microbial fuel cell (MFC) technology. In this study, pyridinic and pyrrolic nitrogen-rich ordered mesoporous carbon (PPN-OMC) synthesized by a facile strategy were successfully applied as cathode catalysts in MFCs. In MFCs with the currently used material, the maximum power density is $1420 \pm 15 \mathrm{~mW} \mathrm{~m}^{-2}$, which is comparable to a conventional platinum catalyst ( $\mathrm{Pt} / \mathrm{C}, 1425 \pm 15 \mathrm{~mW} \mathrm{~m}^{-2}$ ), and the stability of the power output is even better. Mechanism exploration for efficient and stable power generation by electrochemical measurements revealed that the resulting PPN-OMC catalyst displayed superior electrocatalytic activity (nearly $100 \%$ of a four-electron oxygen reduction reaction (ORR) pathway) and durability (nearly no activity change after 100000 potential cycles) for ORR in a neutral phosphate buffer solution (PBS). Further characterization of PPN-OMC implied that the existence of nitrogen substitution with high pyridinic and pyrrolic ratios of $39.8 \%$ and $35.1 \%$ and a large specific surface area of $1160 \mathrm{~m}^{2} \mathrm{~g}^{-1}$ benefitted the electrocatalytic activity and durability. MFCs with the PPN-OMC catalyst produced power much less expensively than those with $\mathrm{Pt} / \mathrm{C}$, indicating that the present PPN-OMC material might be used as an excellent alternative to Pt/C in MFCs.

\section{Introduction}

Energy is one of the biggest challenges in the $21^{\text {st }}$ century, and there is an ever increasing demand for environmental friendly, high power energy sources. In recent years, microbial fuel cells (MFCs), a new kind of biological electric power devices that can recover energy from wastewater, ${ }^{1}$ marine sediment, ${ }^{2}$ and biomass, ${ }^{3}$ have attracted world-wide interests. Similar to the classic fuel cells, MFCs are composed of an anode, on which the fuel is oxidized by microorganisms, and a cathode, which uses the electrons for the reduction of commonly used oxygen. In most cases, the reduction of oxygen on the cathode is catalyzed by the precious platinum (Pt) or it alloys, which largely hampers the large-scale application of MFC technology because of drawbacks such as prohibitive cost, limited supply, and weak durability. ${ }^{4,5}$

${ }^{a}$ State Key Laboratory of Pollution Control and Resources Reuse, School of Environmental Science and Engineering, Tongji University, Shanghai 200092, People's Republic of China. E-mail: yg2chen@yahoo.com; Fax: +86-21-65986313; Tel: +86-21-65981263

${ }^{b}$ Research \& Service Center for Environmental Industry, Yancheng 224051, Jiangsu Province, People's Republic of China

${ }^{c}$ Bohai Drilling Engineering Co., Ltd., China National Petroleum Corporation, Tinajin, 300457, People's Republic of China

$\uparrow$ Electronic supplementary information (ESI) available. See DOI: 10.1039/c7ra00807d
As a result, the global research efforts on MFCs have turned towards the search for a broad range of alternative cathode catalysts based on non-precious metals or their oxides, ${ }^{6}$ microorganisms, ${ }^{7}$ and metal-free doped carbons. ${ }^{8}$ Among these alternative catalysts, metal-free, heteroatom-doped carbon materials have been extensively demonstrated as having the greatest potential due to their excellent electrocatalytic activity and durability as well as the fact that non-precious metal catalysts frequently suffer from dissolution, sintering and agglomeration.

Usually, the heteroatoms used for doping carbon include nitrogen, phosphorus, boron, and sulfur. Among the heteroatomdoped carbon materials, N-doping has been the most popular choice. ${ }^{9}$ The incorporation of $\mathrm{N}$ can enhance the electron-donor property of the carbon matrix and introduce a certain amount of active sites, resulting in an improvement in the ORR activity. Until now, however, the ORR activities of most N-doped carbons were still on a less competitive level compared to Pt catalysts due to insufficient catalytic active sites (functional nitrogen) and a limited surface area, even when the $\mathrm{N}$ content was high. ${ }^{10-12}$ Usually, there are three types of $\mathrm{N}$ atoms in the $\mathrm{N}$-doped carbons, which are pyridinic, pyrrolic, and quaternary $\mathrm{N}$, whereas only the pyridinic and pyrrolic forms have planar structures and have been proven to be active in the ORR. ${ }^{13,14}$ In contrast, the quaternary $\mathrm{N}$ atoms, which usually possess a 3D structure and lead to the low electrical conductivity of $\mathrm{N}$-doped materials due to the interruption of their $\mathrm{p}-\mathrm{p}$ conjugation, are thought to be predominantly responsible for 
the poor catalytic activity. ${ }^{15-17}$ Therefore, the synthesis of $\mathrm{N}$-doped carbon materials with more planar pyridinic and pyrrolic $\mathrm{N}$ atoms and fewer quaternary ones is very important for the preparation of ORR-active catalysts. Recently, nitrogen-doped ordered mesoporous carbon (N-OMC) has been reported to have a large surface area, tunable pore size and huge pore volume, which enhance its ORR activity, and it is being considered as a promising alternative to $\mathrm{Pt} / \mathrm{C}$ for ORR in fuel cell systems. ${ }^{14}$ However, the documented synthesis methods for N-OMC with a relatively larger surface area are complex, ${ }^{18}$ and N-OMC materials with a high content of both pyridinic and pyrrolic $\mathrm{N}$ and a large surface area for more efficient oxygen reduction has seldom been reported.

In this study, the ordered mesoporous metal-free carbon with a high content of both pyridinic and pyrrolic $\mathrm{N}$ and a large surface area (denoted as PPN-OMC for simplicity) prepared by a facile method was employed as a cathode catalyst in microbial fuel cells. The performance of the power output in MFCs with the PPN-OMC catalyst was first evaluated in terms of power density and cell voltage. For comparison, the performance of MFCs with commonly used Pt/C was also investigated. Then, a conceptual explanation of efficient and stable power generation in MFCs with the PPN-OMC catalyst was proposed based on the detailed characterization of the synthesized material and MFCs. This current novel catalyst might offer a new potential for constructing high-performance and less expensive cathodes that are crucial for the large-scale application of MFCs for energy recovery.

\section{Experimental}

\subsection{Preparation of PPN-OMC material}

Conventional ordered mesoporous silica SBA-15 was synthesized using tetraethylorthosilicate (TEOS) and Pluronic P123 according to the method reported previously. ${ }^{19}$ The present carbon material was then prepared by infiltrating the mesopores of $500 \mathrm{mg}$ SBA-15 with $50 \mathrm{mg}$ urea in $10 \mathrm{~mL}$ of a phenol solution at room temperature. After being placed at $80{ }^{\circ} \mathrm{C}$ in a vacuum overnight, the dried mixture was subsequently heated at a temperature rate of $5{ }^{\circ} \mathrm{C} \min ^{-1}$ to $600{ }^{\circ} \mathrm{C}$ for $3 \mathrm{~h}$ under an argon atmosphere. After being carbonized, the silica template was removed by etching in an HF solution (20 wt\%), and the PPN-OMC catalyst was obtained. In this study, all the reagents purchased from Sinopharm Chemical Reagent Co. Ltd. (China) were of analytical grade and were used without further purification, and the solutions were prepared with deionized water.

\subsection{MFC configuration and operation}

The MFC reactors used in this study were membrane-free, single-chamber, air-cathode ones constructed according to the procedure of Logan et al. ${ }^{20}$ The cathodes were made of carbon cloth (30\% wet proofed, BASF), which contained a catalyst loading of $0.5 \mathrm{mg} \mathrm{cm}^{-2}$ on the water-facing side, with four PTFE diffusion layers and one carbon based layer on the air-facing side. The cathode catalysts examined in the present study were the synthesized PPN-OMC material. In order to compare the performance of the as-prepared catalysts with the commonly used cathode catalyst, MFCs with a commercial Pt catalyst ( $20 \%$ of Pt/C, BASF) were set as the blank test. A cathode containing only carbon powder (Vulcan XC-72) was prepared as a non-catalyst control to investigate the electrocatalytic durability of the cathode catalysts in the MFCs. The anodes were carbon fiber brushes that had a two-wire Ti core as the current collector and were treated by the method of Feng et al. ${ }^{21}$

All MFC reactors were inoculated with suspended bacteria from an acetate-fed reactor that had been operating for more than two years and was filled with acetate-laden synthetic wastewater containing $1.0 \mathrm{~L}$ of neutral $50 \mathrm{mM}$ phosphate buffer solution (PBS), $\mathrm{CH}_{3} \mathrm{COONa}, 1000 \mathrm{mg} ; \mathrm{NH}_{4} \mathrm{Cl}, 310 \mathrm{mg} ; \mathrm{KCl}$ $130 \mathrm{mg}$; and minerals and vitamins as described by Lovley and Phillips. ${ }^{22}$ After the voltages in the MFCs were stable, the inoculum was omitted from the solution, and the experimental data were recorded. The external resistance was fixed at $1000 \Omega$ (except as noted), and all the reactors were operated in batch mode at $25 \pm 1{ }^{\circ} \mathrm{C}$.

\subsection{Material and electrochemical analysis}

Scanning electron microscopy (SEM) on a Hitachi S-4800 SEM unit and high-resolution transmission electron (HR-TEM) on a Philips EM-430 TEM unit were applied to investigate the morphology of the as-prepared PPN-PMC materials. Nitrogen adsorption isotherms were measured using a Micromeritics Tristar 3000 analyzer at $77 \mathrm{~K}$. The pore size distribution was obtained from the adsorption branch of the isotherm with the corrected form of the Kelvin equation by means of the Barrett-Joyner-Halenda (BJH) method. The surface area of the PPN-OMC material was determined from the Brunauer-Emmett-Teller (BET) approach. Raman microspectroscopy was performed with a Renishaw inVia unit using a $514.5 \mathrm{~nm}$ Ar ion laser. X-ray diffraction (XRD) patterns were obtained on a Bruker D8 Advance X-ray diffractometer equipped with $\mathrm{Cu} \mathrm{K} \alpha$ radiation. X-ray photoelectron spectroscopic (XPS) analysis was carried out on a Perkin Elmer PHI 5000C ESCA system with a $\mathrm{Mg}$ K-alpha radiation as the exciting source. Spectra obtained over a scan range of $0-1100 \mathrm{eV}$ were recorded and stored using the PHI ACCESS data system and analyzed with XPSPEAK41 software. Before the measurements, all the PPN-OMC samples were degassed overnight at $493 \mathrm{~K}$ under vacuum conditions.

The electrochemical experiments were carried out on a computer-controlled electrochemical workstation (Autolab PGSTAT 302N, Metrohm) in the neutral $50 \mathrm{mM}$ PBS. The threeelectrode cell consisted of an $\mathrm{Ag} / \mathrm{AgCl}$ electrode (3 M KCl-filled) as the reference electrode, a platinum wire as the counter electrode, and a glassy carbon (GC) electrode/rotating ring-disk electrode (RRDE) (Pine Research Instrument Co., USA) loaded with various catalysts as the working electrode. During the preparation of the working electrode, $5.0 \mathrm{mg}$ of the assynthesized catalyst was mixed with $50 \mu \mathrm{L}$ of Nafion solution (5.0\% Nafion in ethanol) and $450 \mu \mathrm{L}$ of deionized water. A $3.0 \mu \mathrm{L}$ suspension of the sonicated mixture was applied onto the GC part of the RRDE, which was successively polished with 1.0 and $0.3 \mu \mathrm{m}$ alumina powder followed by rinsing thoroughly with deionized water, and then fully dried. The catalyst loading was approximately $0.5 \mathrm{mg} \mathrm{cm}^{-2}$. Cyclic voltammogram (CV) 
measurements were carried out to characterize the electrochemical activities on the electrode surface by measuring the current response on the electrode surface to a specific range of potentials with a scan rate of $0.1 \mathrm{~V} \mathrm{~s}^{-1}$. During the RRDE tests, the potential was varied from -0.5 to $0.5 \mathrm{~V} v s$. $\mathrm{Ag} / \mathrm{AgCl}$ at a potential sweep rate of $0.01 \mathrm{~V} \mathrm{~s}^{-1}$. Before the electrochemical measurements, oxygen gas was saturated in the electrolyte by bubbling the gas for $30 \mathrm{~min}$. All electrochemical experiments (except as noted) were carried out at $25 \pm 1{ }^{\circ} \mathrm{C}$.

The electrical conductivities of PPN-OMC and Pt/C were determined at $25 \pm 1{ }^{\circ} \mathrm{C}$ according to the method of Paraknowitsch et al. ${ }^{23}$ Cathode potentials were measured in chronopotentiometry tests by applying a constant current for $60 \mathrm{~min}$ and recording the stable value of the response potential. The catalyst-coated side of the cathode was placed facing the solution, with the uncoated side exposed directly to air. A curve of cathode potentials against current densities was used to evaluate the performance of the cathodes. A higher potential at the same current density indicates a better performance of the cathode catalyst. The internal resistance $\left(R_{\text {in }}\right)$ of the microbial fuel cells (MFCs) was determined by electrochemical impedance spectroscopy (EIS) according to the method of Bard et al. ${ }^{24}$ Impedance measurements were performed with an open circuit voltage (OCV) over a frequency range of 0.05 to $10^{5} \mathrm{~Hz}$ with a sinusoidal perturbation amplitude of $10 \mathrm{mV}$. After the MFCs generated the power stably, the external resistance $\left(R_{\text {ext }}\right)$ was in turn shifted in the range of 10 to $10^{4} \Omega$ to demonstrate the electrochemical polarization and power curves. Data for each resistance were collected after the cells were operated stably for $5 \mathrm{~h}$.

\section{Results and discussion}

\subsection{Performances of MFCs with PPN-OMC as the cathode catalyst}

The cathode catalyst with excellent electrocatalytic activity and durability is one of the urgent needs during the development of MFC technology. The cathode potential of MFCs is usually measured to evaluate the performance of the cathode catalyst, and a higher cathode potential at the same current density indicates a better performance of the cathode catalyst. In this study, the potential of the PPN-OMC cathode in the MFCs (shorten as PPN-OMC-MFCs) was explored and compared to using the Pt/C cathode (Pt/C-MFCs) (Fig. 1). At any current densities, the potentials with PPN-OMC were close to those with $\mathrm{Pt} / \mathrm{C}$. For example, at current densities of 0.19 and $0.57 \mathrm{~mA}$ $\mathrm{cm}^{-2}$, the measured potentials with PPN-OMC were $115 \pm 9$ and $-88 \pm-8 \mathrm{mV}$, respectively, and they were $111 \pm 8$ and $-90 \pm$ $-8 \mathrm{mV}$, respectively, with $\mathrm{Pt} / \mathrm{C}$. The similar cathode potentials suggested that PPN-OMC electrode facilitated the ORR like the $\mathrm{Pt} / \mathrm{C}$ in MFCs.

Then, the performance of the PPN-OMC as a cathode catalyst was evaluated in the MFCs based on the output of the power energy. For comparison, the same investigation was carried out in MFCs equipped with $\mathrm{Pt} / \mathrm{C}$ as the cathode catalyst. The obtained polarization curves and power densities are presented in Fig. 2. The cell voltage generated in the PPN-OMC-MFCs was

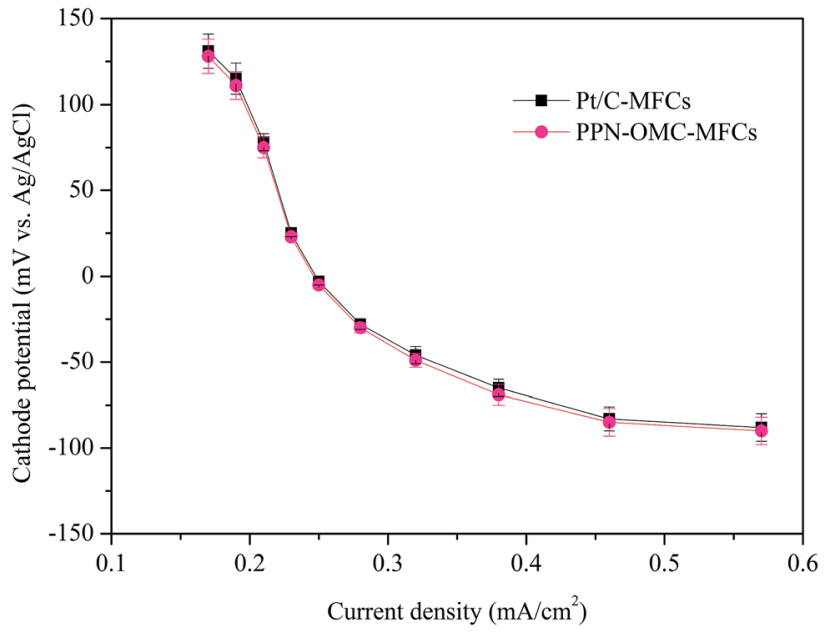

Fig. 1 Comparison of cathode potentials with PPN-OMC and Pt/C at a catalyst loading of $0.5 \mathrm{mg} \mathrm{cm}$. Error bars represent standard deviations of duplicate tests.

$573 \pm 15 \mathrm{mV}$ at an external resistance of $1000 \Omega$, which is close to that in the Pt/C-MFCs $(575 \pm 18 \mathrm{mV})$. Also, as observed in Fig. 2A, the MFCs with PPN-OMC produced a maximal power density of $1420 \pm 15 \mathrm{~mW} \mathrm{~m}^{-2}$, which was almost the same in the Pt/C-MFCs $\left(1425 \pm 15 \mathrm{~mW} \mathrm{~m}^{-2}\right)$. The power output in the PPN-OMC-MFCs was comparable to that in the Pt/C-MFCs. In the previous studies, lots of alternative ORR catalysts, such as transition metals, ${ }^{6-8}$ etc., have attempted to replace or reduce Pt/ $\mathrm{C}$ as the cathode catalyst in MFCs. As far as we know, however, the capacity of power generation in the presence of those catalysts was unsatisfying and much lower than that in the MFCs with Pt/C as the cathode catalyst.

The results of the long-time durability of the two MFCs are presented in Fig. 2B. In the PPN-OMC-MFCs, after operation for 51 days, the maximal power density decreased from $998 \pm 13$ to $925 \pm 10 \mathrm{~mW} \mathrm{~m}^{-2}$, corresponding to about a $7.3 \%$ deterioration. Accordingly, the maximal power density in the Pt/C-MFCs declined from $1008 \pm 15$ to $808 \pm 10 \mathrm{~mW} \mathrm{~m}^{-2}$, a deterioration of more than $19.8 \%$, which was much higher than that in the PPNOMC-MFCs. Typically, the decrease in the power density in the MFCs is due to the deterioration of the electrocatalytic activity of the cathode catalyst and the proton limitation caused by biofilm formation on the cathode surface. In this study, the thicknesses of the biofilms on the cathodes of the PPN-OMCMFCs and Pt/C-MFCs were about $0.1 \mathrm{~cm}$ after 50 days, which might be one of the reasons for the decrease in the maximum power density. However, the drop of the power density in the MFCs without the cathode catalyst (caused by proton limitation only) was found to be about $1.8 \pm 0.2 \%$, revealing that the decrease in the power density in the PPN-OMC-MFCs and Pt/CMFCs was mainly attributed to the deterioration of the electrocatalysts. The stability performance of the power generation in the PPN-OMC-MFCs outperformed that in the Pt/C-MFCs. Overall, the PPN-OMC-MFCs displayed a comparable performance to the Pt/C-MFCs in terms of cell voltage and power density with an even better power generation stability. 

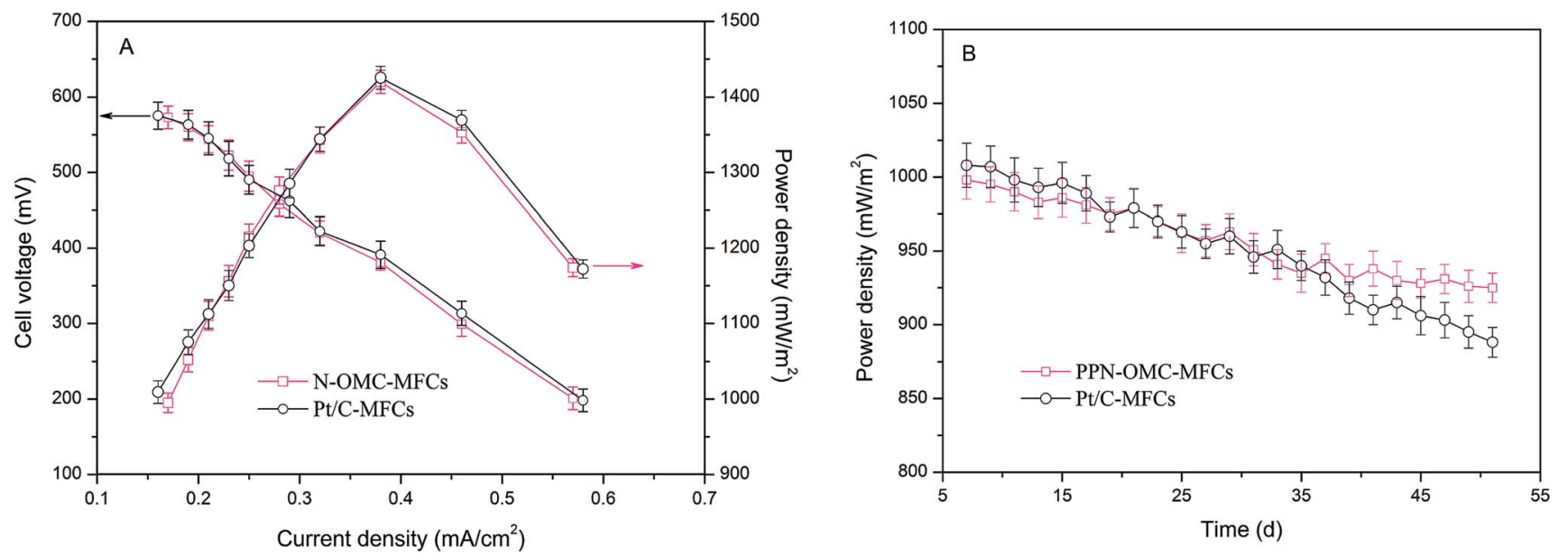

Fig. 2 (A) Power density and cell voltage in the PPN-OMC-MFCs and Pt/C-MFCs. (B) Power density as a function of operation time at $1000 \Omega$. Error bars represent standard deviations of duplicate tests.

\subsection{Understanding PPN-OMC-MFCs for efficient and stable power generation by electrochemical measurements}

In order to understand the electrocatalytic activity and durability of synthesized PPN-OMCs for ORR in neutral media, which is commonly used in bioelectrochemical systems, the CV experiments were conducted by a GC electrode coated with PPNOMC (PPN-OMC/GC) in $\mathrm{O}_{2}$-saturated $50 \mathrm{mM} \mathrm{PBS}(\mathrm{pH} 7.0)$, and GC with a commercial Pt catalyst $(20 \%$ of $\mathrm{Pt} / \mathrm{C}, \mathrm{Pt} / \mathrm{C} / \mathrm{GC})$ was set as the control. As shown in Fig. 3A, both PPN-OMC/GC and Pt/C/ GC exhibited a clear ORR peak in the $\mathrm{O}_{2}$-saturated electrolyte at the potential of 0.15 and $0.18 \mathrm{~V}$. Further comparison of the ORR CVs from PPN-OMC/GC and Pt/C/GC found that their positions, shapes and intensities were similar, demonstrating that like $\mathrm{Pt} /$ $\mathrm{C}$, the synthesized PPN-OMC possessed an excellent electrocatalytic activity for ORR in the neutral electrolyte solution. It is well known that the nitrogen content has a considerable effect on the electrocatalytic activity of the nitrogen-doped carbon catalyst for ORR. Therefore, a nitrogen-doped ordered mesoporous carbon with a lower nitrogen content was created using the same method except that the amount of urea was reduced, and a comparison of the ORR CVs was conducted (Fig. S1†).
Similar trends in the ORR activities were observed based on a series of linear sweep voltammograms (LSV) on a rotating disk electrode (RDE) (Fig. S2 $†$ ). The similar onset potential and ORR current density on PPN-OMC and $\mathrm{Pt} / \mathrm{C}$ electrodes occurred, which was consistent with the CV observations.

To quantify the ORR electron transfer pathway, RRDE measurements for PPN-OMC/GC were performed in the $\mathrm{O}_{2}$ saturated neutral PBS under steady-state conditions, while that for $\mathrm{Pt} / \mathrm{C} / \mathrm{GC}$ served as the contrast. As shown in Fig. 3B, one-step and four-electron pathways for ORR occurred at the Pt/C/GC. Similar to $\mathrm{Pt} / \mathrm{C} / \mathrm{GC}$, the PPN-OMC catalyst also exhibited a one-step process for ORR at an onset potential of about $0.40 \mathrm{~V}$, which was confirmed by the nearly negligible corresponding current for $\mathrm{HO}_{2}{ }^{-}$oxidation recorded at the Pt ring electrode (dash line). According to Bard et al., for a diffusion-controlled process, the transferred electron number $(n)$ per oxygen molecule in the ORR process could be estimated by the RandlesSevcik equation. ${ }^{24}$

$$
i_{\mathrm{p}}=0.4463 n F A C(n F v D / R T)^{1 / 2}
$$
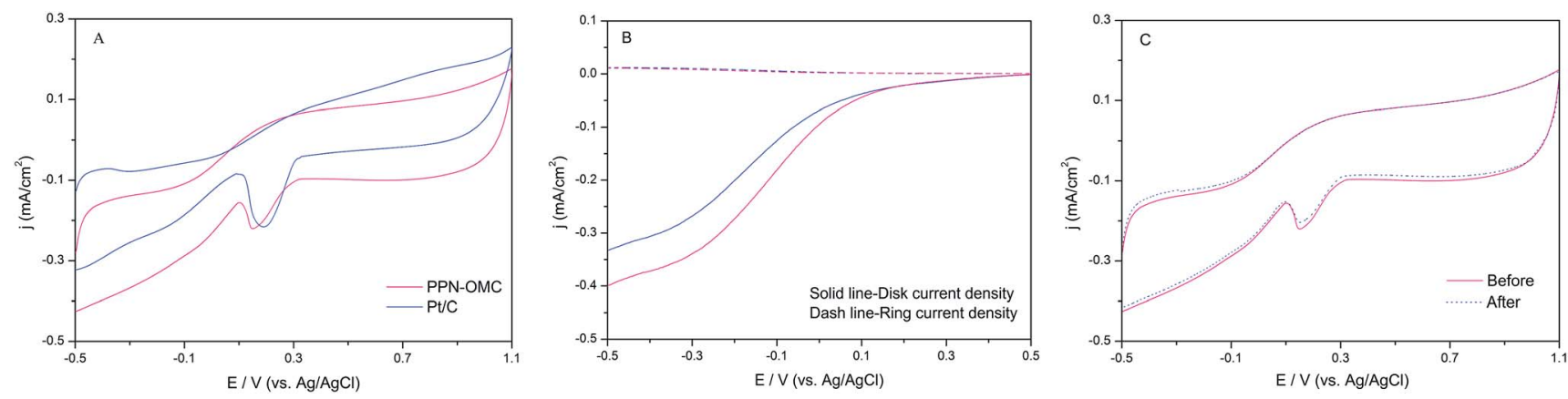

Fig. 3 Electrochemical evaluation. (A) CVs of ORR on PPN-OMC and Pt/C electrodes. (B) RRDE disk and ring currents of PPN-OMC and Pt/C electrodes at a rotation rate of $1200 \mathrm{rpm}$. The Pt ring electrode was poised at $0.5 \mathrm{~V}$. (C) CVs of ORR on PPN-OMC electrode before and after a continuous potentiodynamic sweep for 100000 cycles at $25 \pm 1^{\circ} \mathrm{C}$. The electrolyte used during the voltammogram measurements was the $\mathrm{O}_{2}$-saturated $50 \mathrm{mM}$ PBS solution $(\mathrm{pH} 7.0)$. 
where $n$ is the number of electrons appearing in the halfreaction for the redox couple, $v$ is the potential scanning rate $\left(\mathrm{V} \mathrm{s}^{-1}\right), F$ is Faraday's constant (96 485 $\mathrm{C} \mathrm{mol}^{-1}$ ), $A$ is the electrode area $\left(\mathrm{cm}^{2}\right), R$ is the universal gas constant $\left(8.314 \mathrm{~J}\left(\mathrm{~mol}^{-1}\right.\right.$ $\left.\mathrm{K}^{-1}\right)$ ), $T$ is the absolute temperature (K), which is $298.15 \mathrm{~K}$ in this study, and $D$ is the analyte's diffusion coefficient $\left(\mathrm{cm}^{2} \mathrm{~s}^{-1}\right)$. According to the data shown in Fig. 4 and eqn (1), the $n$ of the PPN-OMC material prepared in this study was calculated to be about 3.75, indicating that a four-electron pathway for ORR was nearly obtained for the PPN-OMC catalyst in the neutral PBS. The voltammetry measurements revealed that the current PPNOMC material had an excellent ORR activity in the commonly used biological electrolyte.

Also, continuous potential cycling was performed to investigate the electrocatalytic durability of the current PPN-OMC catalyst towards ORR. As can be seen in Fig. 3C, almost no obvious decrease in the current density occurred after 100000 continuous cycles between $-0.5 \mathrm{~V}$ and $1.0 \mathrm{~V}$ in the $\mathrm{O}_{2}$-saturated PBS electrolyte. The electrocatalytic durability of the PPN-OMC catalyst for ORR was superior. When used in the bioelectrochemical systems, the metabolite tolerance ability is a crucial feature of catalysts. Thus, in the present study, the crossover behavior of the synthesized PPN-OMC was examined by adding $5 \mathrm{mM}$ sodium formate, a typical metabolite in bioelectrochemical reactors, to the ORR system (Fig. S3†). It was observed that the ORR current density remained largely unchanged after the addition of sodium formate, which suggested that the PPN-OMC catalyst possessed an excellent tolerance to the metabolite crossover effect. The as-prepared PPNOMC material might be a perfect electrocatalyst with a high metabolite tolerance ability when applied in biological systems, although the tests of the tolerance ability of PPN-OMC for other metabolites, such as sulphide and methanol, still need to be conducted.

It has been reported in the literature that the internal resistance plays an important role during the generation of power energy in MFCs. ${ }^{20}$ In the present investigation, therefore, the internal resistance of MFCs with different catalysts was investigated based on the EIS analysis. It was found that the $R_{\text {in }}$ of MFCs with PPN-OMC and Pt/C were $89 \pm 2$ and $85 \pm 3 \Omega$, respectively. Usually, the internal resistance of a MFC reactor consists of two parts: the electrolyte ohmic loss due to the movement of electrons through the electrolyte, and the electrode ohmic loss caused by the movement of electrons through the electrode and wires. In this study, due to the fact that the two MFC reactors had the same configuration, such as the anode material, electrode distance, etc., the difference in the $R_{\text {in }}$ between the PPN-OMC-MFCs and Pt/C-MFCs was derived from the diversity of the electrical characteristics, especially the conductivity, between the synthesized PPN-OMC and Pt/C. The electrical conductivities of the PPN-OMC and Pt/C cathodes were measured to be about $40 \pm 2$ and $45 \pm 2 \mathrm{~S} \mathrm{~cm}^{-1}$, respectively. The lower conductivity of the PPN-OMC cathode resulted in the slightly higher $R_{\text {in }}$ of the PPN-OMC-MFCs. In this case, the power generation performance was analogous to that in the Pt/C-MFCs.

\subsection{Understanding PPN-OMC activity and durability by surface chemistry characterization}

The morphology and structure arrangements of the obtained PPNOMC sample were observed by SEM and TEM. The PPN-OMC material showed a rod-like morphology (Fig. 4A and B), and its
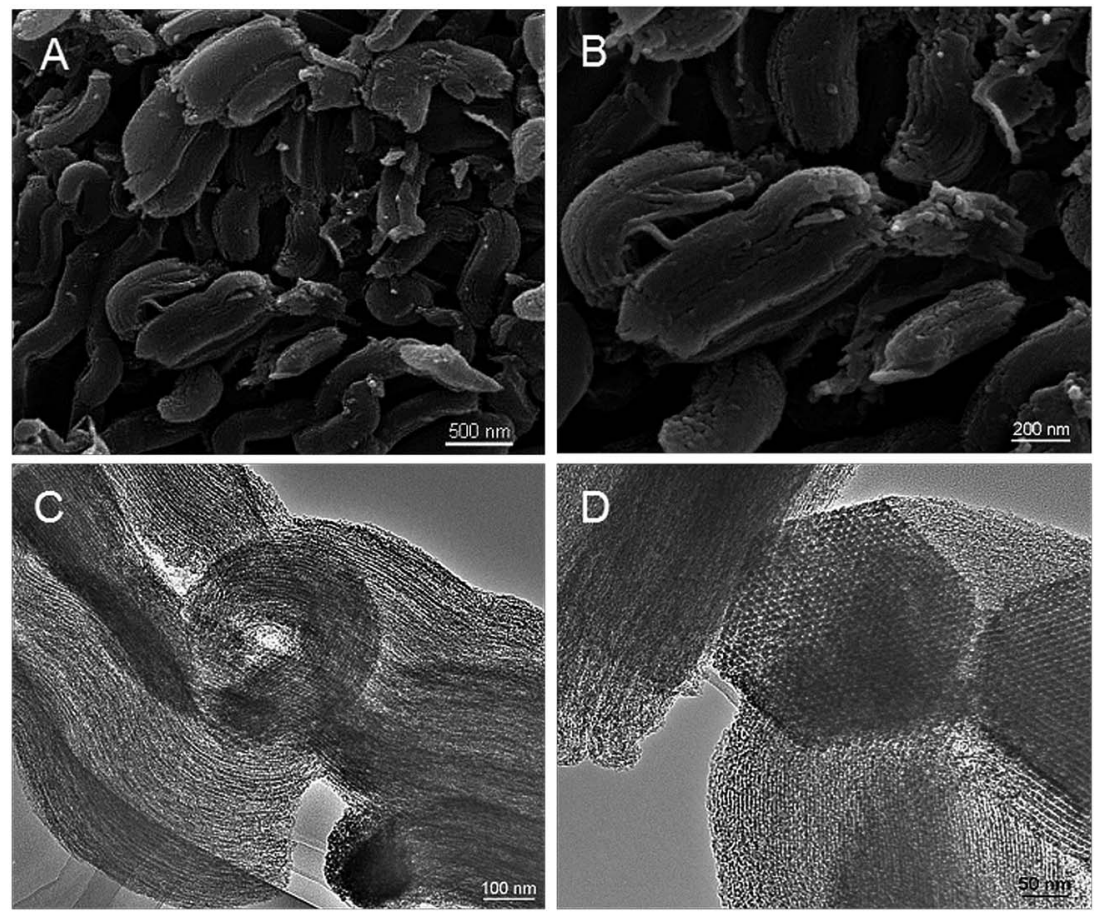

Fig. 4 ( $A$ and $B$ ) SEM and (C and D) HR-TEM images of the synthesized PPN-OMC material. 
average particle size was about $1.0 \mu \mathrm{m}$ long, which was similar to the original silica template. Further investigation found that the PPN-OMC sample exhibited bundles of well-ordered isotropic channels, showing that the PPN-OMC product still retained the original character of the silica template. HR-TEM images of the current PPN-OMC are shown in Fig. 4C and D. The PPN-OMC material consisted of well-ordered hexagonal arrays of mesopores with one-dimensional channels. Also, the cross-sectional HR-TEM images clearly display a honeycomb-like arrangement of the mesopores, implying that the hexagonal mesostructure was preserved after the high-temperature carbonization and $\mathrm{HF}$ dissolution.

The nitrogen adsorption-desorption isotherms of the PPNOMC material are shown in Fig. 5A. Type IV curves with a sharp capillary condensation step and a $\mathrm{H}_{1}$-type hysteresis loop were observed for the as-obtained carbon materials, which is indicative of uniform cylindrical pores. As shown in the inset of Fig. 5A, a narrow pore size distribution with a mean value of $3.8 \mathrm{~nm}$ was calculated from the adsorption branch using the $\mathrm{BJH}$ model, and a specific surface area of $1160 \mathrm{~m}^{2} \mathrm{~g}^{-1}$ was exhibited. In the literature, the reported specific surface area of PPN-OMC with a high content of nitrogen, especially pyridinic and pyrrolic nitrogen, was usually less than $1000 \mathrm{~m}^{2} \mathrm{~g}^{-1,23,25,26}$ indicating that the current strategy for PPN-OMC synthesis benefitted the improvement of the specific surface area. Usually, a PPN-OMC catalyst with a larger surface area holds more active sites to be exposed to oxygen molecules and benefits the improvement of ORR activity.

The XRD patterns were applied to characterize the structure of the mesoporous carbon, and the results are shown in Fig. 5B. The high angle region (above $2 \theta=10^{\circ}$ ) of the PPN-OMC samples via the current process exhibited peaks at $2 \theta=26$ and $44^{\circ}$, which can be ascribed to (002 and 101) diffraction peaks from the graphitic pore walls, respectively. The XRD patterns indicated that the PPN-OMC sample possessed graphitic (crystalline) rather than amorphous pore walls. The small angle XRD pattern of the synthesized PPN-OMC sample is shown in the inset of Fig. 5B. A high intensity diffraction peak of (100) and two additional well-resolved diffraction peaks of (110) and (200) occurred, which can be assigned to a $2 \mathrm{D}$ hexagonal lattice, similar to that of the SBA-15 template, demonstrating that the mesoporous structure was retained even after the high temperature carbonization for the formation of graphitic pore walls.

To further evaluate the structure and quality of the PPN-OMC material, Raman spectroscopy measurements were taken on a Renishaw Raman spectroscope, and a typical Raman spectrum is displayed in Fig. 5C. Three main peaks were assigned, D band $\left(\sim 1316 \mathrm{~cm}^{-1}\right)$, $\mathrm{G}$ band $\left(\sim 1594 \mathrm{~cm}^{-1}\right)$ and $2 \mathrm{D}$ band $(\sim 2653$ $\mathrm{cm}^{-1}$ ). The $\mathrm{G}$ band showed a blue-shift compared to the pristine carbon black (data not shown here), which was a sign of atomic insertions. Usually, the intensity ratio of the $\mathrm{D}$ to $\mathrm{G}$ band $\left(I_{\mathrm{D}} / I_{\mathrm{G}}\right)$ can give qualitative information on the degree of the defects, and a higher value indicates more defects present in the carbon materials. According to the Raman spectrum, the $I_{\mathrm{D}} / I_{\mathrm{G}}$ ratio of PPN-OMC in this study was about 1.25, implying a high degree of defects in the crystalline structure of the PPN-OMC sample.

XPS measurements were performed to probe the chemical composition and nitrogen content in the PPN-OMC material. The survey scan spectrum from the XPS analysis for PPN-OMC revealed the presence of $\mathrm{C} 1 \mathrm{~s}, \mathrm{O} 1 \mathrm{~s}$ and $\mathrm{N} 1 \mathrm{~s}$ without any other impurities, showing that the current PPN-OMC material was completely metal-free (Fig. 6A). In the spectrum of PPNOMC, the peaks at 285.8, 399.6, and $537.2 \mathrm{eV}$ corresponded to $\mathrm{C} 1 \mathrm{~s}$ of $\mathrm{sp}^{2} \mathrm{C}, \mathrm{N} 1 \mathrm{~s}$, and $\mathrm{O} 1 \mathrm{~s}$ of the absorbed oxygen, respectively, and the content of carbon, nitrogen and oxygen were measured to be about $80.0 \%, 16.1 \%$ and $3.9 \%$, respectively. Generally, the reported nitrogen content in the PPN-OMC sample was less than $15.0 \%,{ }^{27-29}$ suggesting that the present strategy for PPNOMC synthesis benefited from the improvement of the nitrogen content. As PPN-OMC materials with a high nitrogen content have been demonstrated to show better physiochemical properties, such as electrocatalytic activity, electrical conductivity, etc. ${ }^{14,28,29}$ the PPN-OMC prepared in this study might have a wide range of practical applications.

The high resolution XPS N 1s spectrum of the current PPNOMC material is shown in Fig. 6B. It was found that the spectrum of $\mathrm{N} 1 \mathrm{~s}$ had three components, indicating that the $\mathrm{N}$ atoms were in three different bonding situations. The $\mathrm{N}$ peaks located at around 399.3, 400.5 and $401.8 \mathrm{eV}$ corresponded to the pyridinic, pyrrolic, and graphite nitrogen atoms, respectively. It is well known that the pyridinic and pyrrolic nitrogen are a kind of $\mathrm{N}$ that contribute one p-electron to the aromatic $\pi$-system, and they have a lone electron pair in the plane of the carbon matrix,
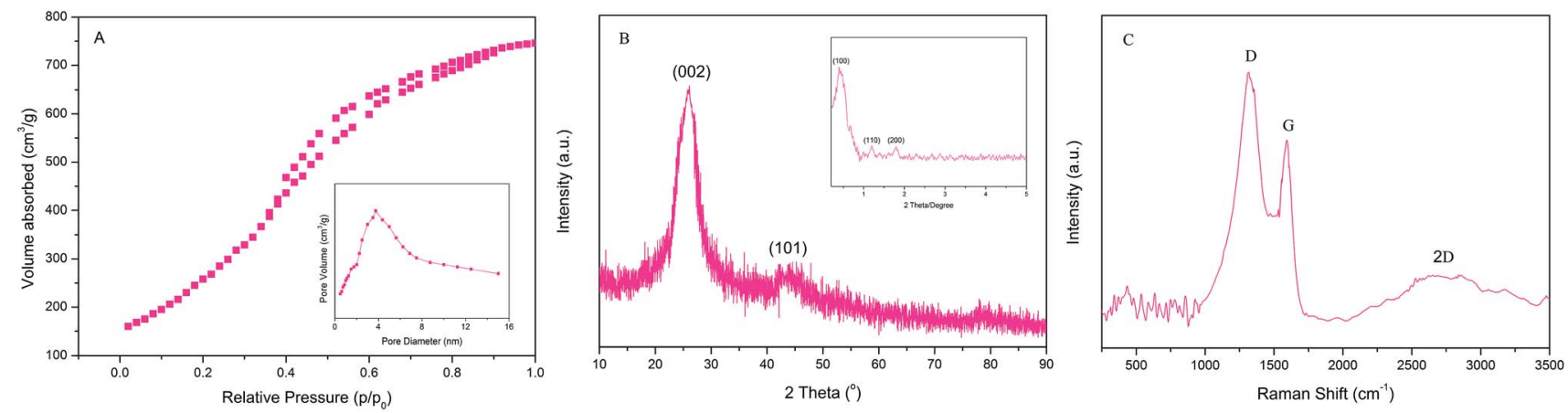

Fig. 5 (A) Nitrogen sorption isotherms and pore size distribution (inset) of PPN-OMC materials. (B) XRD patterns of the synthesized PPN-OMC. (C) Raman spectrum of PPN-OMC materials showing a high peak intensity ratio of $D$ to $G$ band. 

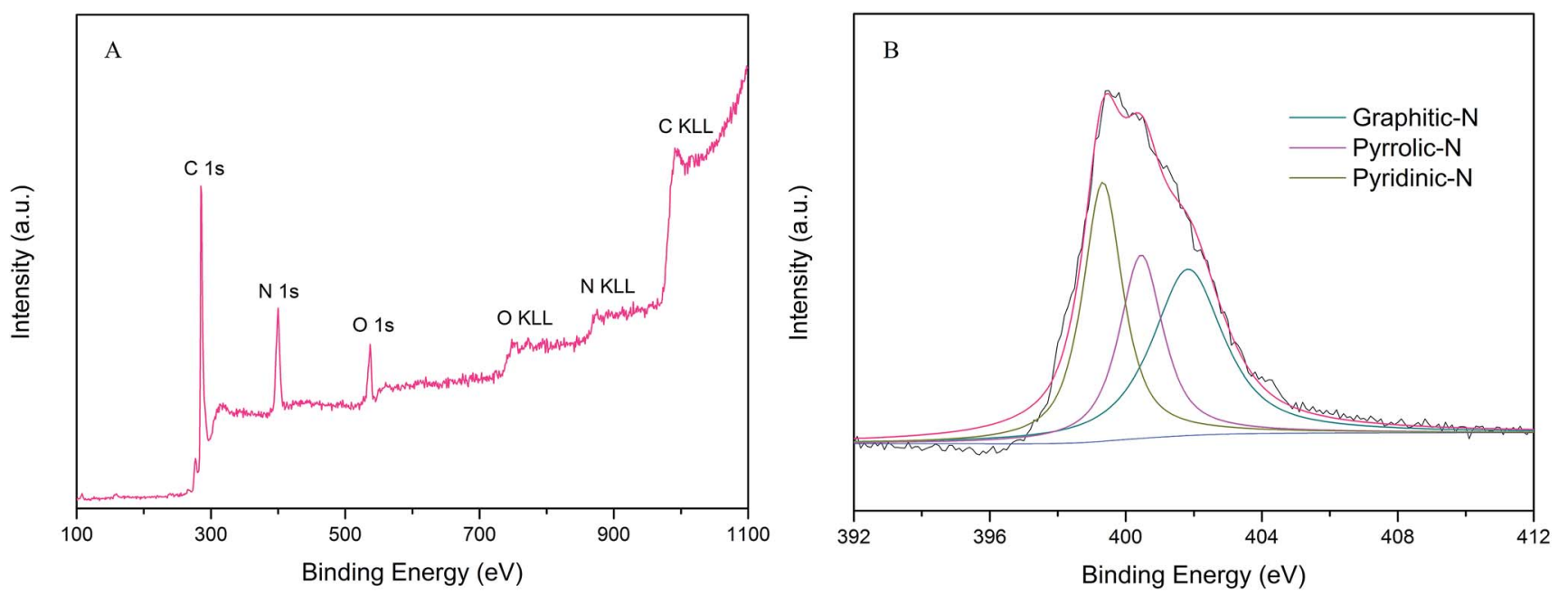

Fig. 6 (A) XPS survey for the PPN-OMC material and (B) high-resolution N 1s spectrum.

which can increase the electron-donor property of materials. The graphite nitrogen usually represents the $\mathrm{N}$ atoms replacing the $\mathrm{C}$ atoms in the structure of the carbon material. In the $\mathrm{N}$ peak of the current PPN-OMC material, the content of pyridinic, pyrrolic, and graphite nitrogen was calculated to be $39.8 \%$, $35.1 \%$ and $25.1 \%$ of the total one, which suggested that pyridinic and pyrrolic $\mathrm{N}$ dominated the $\mathrm{N}$ content of the asprepared PPN-OMC material. In the structure of the nitrogendoped carbon materials, the functional nitrogen responsible for ORR usually corresponds to the pyridinic and pyrrolic ones because they are obtained by doping at the edge of the carbon materials, whereas the graphite $\mathrm{N}$ is the result of in-plane doping. ${ }^{30-35}$ It is well known that there are three styles for $\mathrm{O}_{2}$ adsorption on the electrode surface for ORR, i.e. Griffith model, Pauling model and Yeager model. ${ }^{36}$ Meanwhile, the doping of pyridinic and pyrrolic $\mathrm{N}$ into graphitic sheets at the edge has been found to induce the charge delocalization of the carbon atoms, which changes the chemisorption model of $\mathrm{O}_{2}$ during the oxygen reduction. ${ }^{32}$ Thus, the following observed electrocatalytic activity and durability of the PPN-OMC catalyst was mainly due to the pyridinic and pyrrolic $\mathrm{N}$-induced charge delocalization, which changed the chemisorption model of $\mathrm{O}_{2}$ from the usual end-on adsorption (Pauling model) to the sideon one (Yeager model), and it effectively weakened the $\mathrm{O}-\mathrm{O}$ to facilitate the oxygen reduction on the PPN-OMC electrode..$^{31,32}$

The detailed C 1s spectrum of PPN-OMC ranging from 280 to $295 \mathrm{eV}$ is shown in Fig. S4. $\dagger$ Generally, there were three different $\mathrm{C}$ groups in the XPS spectrum of PPN-OMC, which were characterized by the observation of spectral peaks: $\mathrm{C}-\mathrm{C}$ at $285.2 \mathrm{eV}$, $\mathrm{C}-\mathrm{O}$ at $286.5 \mathrm{eV}$, and $\mathrm{O}-\mathrm{C}=\mathrm{O}$ at $288.6 \mathrm{eV}$. Additionally, an obvious $\mathrm{O} 1 \mathrm{~s}$ peak at $536.8 \mathrm{eV}$ occurred for the synthesized PPNOMC, possibly due to the adsorbed moisture, atmospheric $\mathrm{O}_{2}$, or $\mathrm{CO}_{2}$, as well as the residual oxygen-containing groups, such as carbonyl and carboxyl groups. The apparent $\mathrm{O} 1$ s peak was an additional advantage for the current PPN-OMC as catalysts of the ORR electrode because the nitrogen-doped carbon material with a higher $\mathrm{O} 1 \mathrm{~s}$ content has a stronger ability for $\mathrm{O}_{2}$ adsorption, which is very important for ORR. ${ }^{34}$

Based on the above discussion, the main pathways and mechanisms of oxygen reduction in PPN-OMC-MFCs with

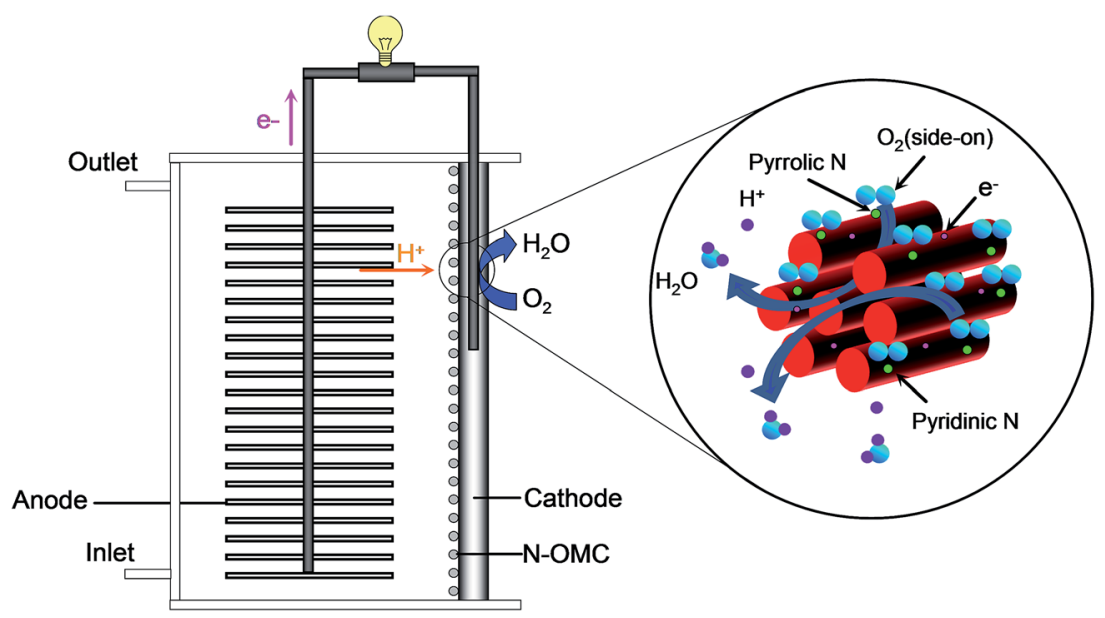

Fig. 7 Schematic pathway and mechanism of oxygen reduction in PPN-OMC-MFCs with neutral PBS. 
neutral PBS as an electrolyte are proposed in Fig. 7. A fourelectron pathway of the PPN-OMC catalyst for ORR in neutral PBS is favored by the side-on configuration rather than the endon one due to the fact that doping ordered mesoporous carbon with pyridinic and pyrrolic nitrogens and a large surface area in the PPN-OMC electrode created large amounts of metal-free active sites for efficient oxygen reduction, which was one of the most important reasons for the excellent electrocatalytic activity and durability of the PPN-OMC for ORR exhibited in MFCs.

\subsection{Implications of PPN-OMC-MFCs for practical application based on cost analysis}

As mentioned above, the practical application of MFCs is largely limited by the high cost of the cathode catalyst, especially as the scale increases. Thus, it is necessary to evaluate the cost of the PPN-OMC catalyst when being applied as a cathode catalyst in MFCs. A schematic illustration of the fabrication approach for the PPN-OMC catalyst is shown in Fig. S5. $\dagger$ It can be seen that the mentioned synthesis method for the PPN-OMC was facile and allowed a massive yield. At present, the prices of SBA-15, urea and phenol are \$3.3, 0.004 and $0.09 / \mathrm{g}$, respectively, and the reagent cost per gram for PPN-OMC in this study was approximately $\$ 1.6((3.3 \times 0.5+$ $0.004 \times 0.05+0.09 \times 10 \times 5 \%) /(0.5+0.05+0.5)=1.6)$. Usually, the conventional $\mathrm{Pt} / \mathrm{C}$ is prepared with dihydrogen hexachloroplatinate (IV) hexahydrate and carbon supports by complex techniques with impregnation and reduction. ${ }^{37}$ Currently, the price of dihydrogen hexachloroplatinate (IV) hexahydrate with a Pt content of $37.5 \%$ is about $\$ 212.1 / \mathrm{g}$, and the reagent cost for Pt/C containing $1 \mathrm{~g}$ Pt is $\$ 565.6$ (214.4/ $37.5 \%=565.6$ ) when only the cost of the Pt salt was taken into account. Obviously, the reagent cost for the PPN-OMC catalyst synthesized in this study was much lower than that of $\mathrm{Pt} / \mathrm{C}$ (\$1.6 versus \$565.6). In light of the complex process for $\mathrm{Pt} / \mathrm{C}$ preparation, the PPN-OMC synthesized with the facile method is anticipated to be much cheaper than the conventional $\mathrm{Pt} / \mathrm{C}$, and the power output per cost in the PPN-OMCMFCs would be much higher than that in Pt/C-MFCs.

\section{Conclusions}

In summary, nitrogen-doped, ordered mesoporous carbon with a high content of both pyridinic and pyrrolic nitrogens and a large specific surface area was synthesized via a simple method, and it was demonstrated to possess excellent electrocatalytic activity and durability for oxygen reduction in a neutral electrolyte. When the current PPN-OMC was applied as a metalfree catalyst for cathode ORR in MFCs, the power generation performance was almost the same as or even better than that of the conventional Pt catalyst. The PPN-OMC material might be an ideal alternative to Pt catalysts in MFCs with a long run. This novel cathode catalyst offers a new potential for constructing high-performance and less expensive cathodes that are crucial for the large-scale application of MFCs and other bioelectrochemical technologies.

\section{Acknowledgements}

Financial support from the National Natural Science Funds for Distinguished Young Scholar (No. 51425802), National Science Foundation of China (No. 51108332 and 51208371), State Key Laboratory of Pollution Control and Resource Reuse Foundation (No. PCRRK16003), Fundamental Research Funds for the Central Universities, Chinese Polar Environment Comprehensive Investigation and Assessment Programmers (No. CHINARE2016-0201), and Cooperative Innovation Fund of Jiangsu Province (No. BY2014114 and BY2014115) is appreciated.

\section{References}

1 U. Schröder, F. Harnisch and L. T. Angenent, Energy Environ. Sci., 2015, 8, 513-519.

2 S. B. Quek, L. Cheng and R. Cordruwisch, Water Res., 2015, 77, 64-71.

3 A. E. Inglesby and A. C. Fisher, Energy Environ. Sci., 2012, 5, 7996-8006.

4 C. Santoro, F. Soavi, A. Serov, C. Arbizzani and P. Atanassov, Biosens. Bioelectron., 2015, 78, 229-235.

5 C. Santoro, A. Serov, L. Staryha, M. Kodali, J. Gordon, S. Babanova, O. Bretschger, K. Artyushkova and P. Atanassov, Energy Environ. Sci., 2016, 9, 2346-2353.

6 R. Burkitt, T. R. Whiffen and E. H. Yu, Appl. Catal., B, 2016, 181, 279-288.

7 Y. Tao, Q. Liu, J. Chen, W. Bo, Y. Wang, L. Ke, M. Li, H. Jiang, Z. Lu and W. Dong, Environ. Sci. Technol., 2016, 50, 78897895.

8 M. Shao, Q. Chang, J. P. Dodelet and R. Chenitz, Chem. Rev., 2016, 116, 3594-3657.

9 Y. J. Wang, D. P. Wilkinson and J. Zhang, Chem. Rev., 2011, 111, 7625-7651.

10 C. H. Choi, S. H. Park and S. I. Woo, ACS Nano, 2012, 6, 70847091.

11 R. Silva, D. Voiry, M. Chhowalla and T. Asefa, J. Am. Chem. Soc., 2013, 135, 7823-7826.

12 Y. Zhao, L. Yang, S. Chen, X. Wang, Y. Ma, Q. Wu, Y. Jiang, W. Qian and Z. Hu, J. Am. Chem. Soc., 2013, 135, 1201-1204.

13 X. Cui, S. Yang, X. Yan, J. Leng, S. Shuang, P. M. Ajayan and Z. Zhang, Adv. Funct. Mater., 2016, 26, 5708-5717.

14 S. Yang, X. Feng, X. Wang and K. Mullen, Angew. Chem., Int. Ed. Engl., 2011, 50, 5339-5343.

15 J. Casanovas, J. M. Ricart, J. Rubio, F. Illas and J. M. JiménezMateos, J. Am. Chem. Soc., 1996, 118, 8071-8076.

16 T. B. Martins, R. H. Miwa, A. J. da Silva and A. Fazzio, Phys. Rev. Lett., 2007, 98, 304-308.

17 X. B. Wang, Y. Q. Liu, D. B. Zhu, L. Zhang, H. Z. Ma, N. Yao and B. L. Zhang, J. Phys. Chem. B, 2002, 106, 2186-2190.

18 G. P. Mane, S. N. Talapaneni, C. Anand, S. Varghese, H. Iwai, Q. Ji, K. Ariga, T. Mori and A. Vinu, Adv. Funct. Mater., 2012, 22, 3596-3604.

19 T.-W. Kim, I.-S. Park and R. Ryoo, Angew. Chem., 2003, 115, 4511-4515.

20 B. E. Logan and J. M. Regan, Environ. Sci. Technol., 2006, 40, 5172-5180. 
21 Y. Feng, Q. Yang, X. Wang and B. E. Logan, J. Power Sources, 2010, 195, 1841-1844.

22 D. R. Lovley and E. J. Phillips, Appl. Environ. Microbiol., 1988, 54, 1472-1480.

23 J. P. Paraknowitsch, J. Zhang, D. Su, A. Thomas and M. Antonietti, Adv. Mater., 2010, 22, 87-92.

24 A. J. Bard and L. R. Faulkner, Electrochemical Methods, Wiley, New York, 2nd edn, 2001.

25 N. Liu, L. Yin, C. Wang, L. Zhang, N. Lun, D. Xiang, Y. Qi and R. Gao, Carbon, 2010, 48, 3579-3591.

26 J. Wei, D. Zhou, Z. Sun, Y. Deng, Y. Xia and D. Zhao, Adv. Funct. Mater., 2013, 23, 2322-2328.

27 J. Wang, R. Ma, Y. Zhou and Q. Liu, J. Mater. Chem. A, 2015, 3, 12836-12844.

28 J. S. Lee, X. Wang, H. Luo, G. A. Baker and S. Dai, J. Am. Chem. Soc., 2009, 131, 4596-4597.

29 W. Li, D. Chen, Z. Li, Y. Shi, Y. Wan, J. Huang, J. Yang, D. Zhao and Z. Jiang, Electrochem. Commun., 2007, 9, 569573.
30 G. Yang, W. Choi, X. Pu and C. Yu, Energy Environ. Sci., 2015, 8, 1799-1807.

31 L. Feng, L. Yang, Z. Huang, J. Luo, M. Li, D. Wang and Y. Chen, Sci. Rep., 2013, 3, 442-455.

32 K. Gong, F. Du, Z. Xia, M. Durstock and L. Dai, Science, 2009, 323, 760-764.

33 S. Kundu, T. C. Nagaiah, W. Xia, Y. Wang, S. V. Dommele, J. H. Bitter, M. Santa, G. Grundmeier, M. Bron and W. Schuhmann, J. Phys. Chem. C, 2009, 113, 14302-14310.

34 L. Qu, Y. Liu, J.-B. Baek and L. Dai, ACS Nano, 2010, 4, 13211326.

35 C. V. Rao, C. R. Cabrera and Y. Ishikawa, J. Phys. Chem. Lett., 2010, 1, 2622-2627.

36 E. Yeager, Electrochim. Acta, 1984, 29, 1527-1537.

37 B. Xue, P. Chen, Q. Hong, J. Lin and K. L. Tan, J. Mater. Chem., 2001, 11, 2378-2381. 